\title{
The Motion Analysis of Multi-degree-of-freedom Assembly System in Space Station
}

\author{
Wang Pengfei ${ }^{1,2, *}$, Zhang Chengli ${ }^{1,2}$, Liu Tonghui ${ }^{1,2}$, Xing Shuai $^{1,2}$ \\ ${ }^{1}$ Beijing Institute of Spacecraft Environment Engineering, Beijing, China \\ ${ }^{2}$ Beijing Engineering Research Center of the Intelligent Assembly Technology and Equipment for Aerospace Product, Beijing, China
}

\section{Email address:}

wangpengfeimisha@163.com (Wang Pengfei), zfox82@163.com (Zhang Chengli)

\section{To cite this article:}

Wang Pengfei, Zhang Chengli, Liu Tonghui, Xing Shuai. The Motion Analysis of Multi-degree-of-freedom Assembly System in Space Station. Science Discovery. Vol. 6, No. 1, 2018, pp. 62-71. doi: 10.11648/j.sd.20180601.21

Received: January 13, 2018; Accepted: February 27, 2018; Published: May 23, 2018

\begin{abstract}
Spacecraft assembly is the final stage and the key link in the realization of functionality and performance of aerospace products, through the introduction of multi-degree-of-freedom assembly system in the intelligent assembly process of the large space station, the intelligent assembly of large-scale products in the sealed capsule is realized. In this paper, based on three elements, those are the spacecraft, the product to be installed and the multi-degree-of-freedom assembly system, a motion model of the multi-degree-of-freedom assembly system is established, the movement trajectory of multi-degree-of-freedom assembly system is analyzed detailly, according to the principle of inverse kinematics, path planning of the multi-degree-of-freedom assembly system is carried out. Through the simulation software, the trajectory and path of the multi-degree-of-freedom assembly system are simulated, the assembly path of the product in the sealed space of the space station is obtained. Finally, by constructing the virtual environment of spacecraft assembly, the theoretical model and the calculation results are verified. Through analyzing the path planning of multi-degree-of-freedom assembly system in space station, intelligent assembly of spacecraft products is achieved.
\end{abstract}

Keywords: Space Station, Assembly System, Motion Track, Path Planning

\section{空间站多自由度装配系统运动分析}

王鹏飞 ${ }^{1,2 *}$, 张成立 ${ }^{1,2}$, 刘同辉 ${ }^{1,2}$, 邢帅 ${ }^{1,2}$

${ }^{1}$ 北京卫星环境工程研究所, 北京, 中国

2 北京市航天产品智能装配技术与装备工程技术研究中心, 北京, 中国

邮箱

wangpengfeimisha@163.com（王鹏飞）,zfox82@163.com（张成立）

摘要: 航天器装配是航天产品功能和性能实现的最终阶段和关键环节, 在超大空间站智能装配过程中引入多自由度装 配系统实现密封舱内大型产品的智能装配。本文以航天器、待安装产品以及多自由度装配系统三要素为基础, 建立了 多自由度装配系统的运动模型, 详细分析了多自由度装配系统的运动轨迹, 并根据逆运动学原理对多自由度装配系统 进行路径规划。采用仿真软件对多自由度装配系统的运动轨迹及路径进行模拟, 得到空间站超大航天器密封舱内产品 的装配路径, 最后构建航天器装配的虚拟环境, 通过虚拟仿真来验证理论模型及计算结果。空间站多自由度装配系统 的运动分析实现了航天器产品智能装配。

关键词: 空间站, 装配系统, 运动轨迹, 路径规划 


\section{1. 引言}

总体装配是航天产品功能和性能实现的最终阶段和 关键环节, 是影响航天器研制质量和服役性能的重要因素, 航天产品总装技术与装备水平直接影响到航天产品研制 的质量、效率，甚至产品的成败[1] [3]。目前，航天产品 装配主要依赖人工操作, 航天器外部产品尚可以辅以吊具 等简单工艺装备进行定位和支撑, 而航天器内部产品的装 配, 由于内部空间限制, 产品装配极其困难, 装配质量严 重依赖作业人员。随着空间站项目建设, 产品功能趋于集 成化和标准化, 设备的结构尺寸、质量特性均超过了以往 载人航天器, 存在多种大重量载荷产品, 最重的产品达到 了 $500 \mathrm{~kg}$ [4] [6]。不同产品结构形式各异, 安装状态也各 不相同, 传统简单通过人工托举或起吊的方式已无法满足 空间站舱内大重量产品的总装需求, 亟需引入新型工艺装 备与装配技术。

针对空间站型号研制过程中大重量产品多、工况变化 复杂、位姿调整精度高等特点, 尤其是密封舱内大尺寸、 大重量产品复杂工况下的安装, 为了满足空间站型号总装 的需求, 设计了多自由度装配系统, 该系统具有 8 个自由 度, 可以实现重量达到 $500 \mathrm{~kg}$ 的产品及多种不同安装角度 产品的精确定位装配。
本文以航天器、待安装产品以及多自由度装配系统三 要素为基础, 建立了多自由度装配系统的运动模型, 详细 分析了多自由度装配系统的运动轨迹, 并通过仿真分析验 证了运动模型, 实现了空间站大型产品装配的精确定位。

\section{2. 多自由度装配系统简介}

空间站多自由度装配系统结构形式为串联伸缩机械 臂, 主体横向移动、垂直伸缩、未端垂直伸缩、水平伸缩、 偏移、回转、俯仰、偏航 8 个自由度, 主体结构包含移动 平台与串联伸缩机构组成。

多自由度装配系统首先通过移动平台实现串联伸缩 机构及产品在不同总装位置间的快速移动, 并能够提供稳 定的支撑。多自由度串联伸缩机构安装在移动平台上方, 通过末端机构拾取产品, 通过底部平台移动到航天器指定 位置, 水平伸缩机构将产品运送到舱内指定区域, 然后通 过偏移机构及垂直伸缩机构将产品移动到安装位置, 最后 通过回转、俯仰、偏航调节机构将产品进行精确定位安装, 定位过程同时需要微调垂直伸缩、水平伸缩、偏移机构。 对于复杂的多角度产品装配还需要调节横向移动机构, 避 免由于末端垂直伸缩行程不足导致无法装配的问题。

多自由度装配系统的结构形式如图1所示, 多自由度 装配系统与航天器装配关系如图2所示。

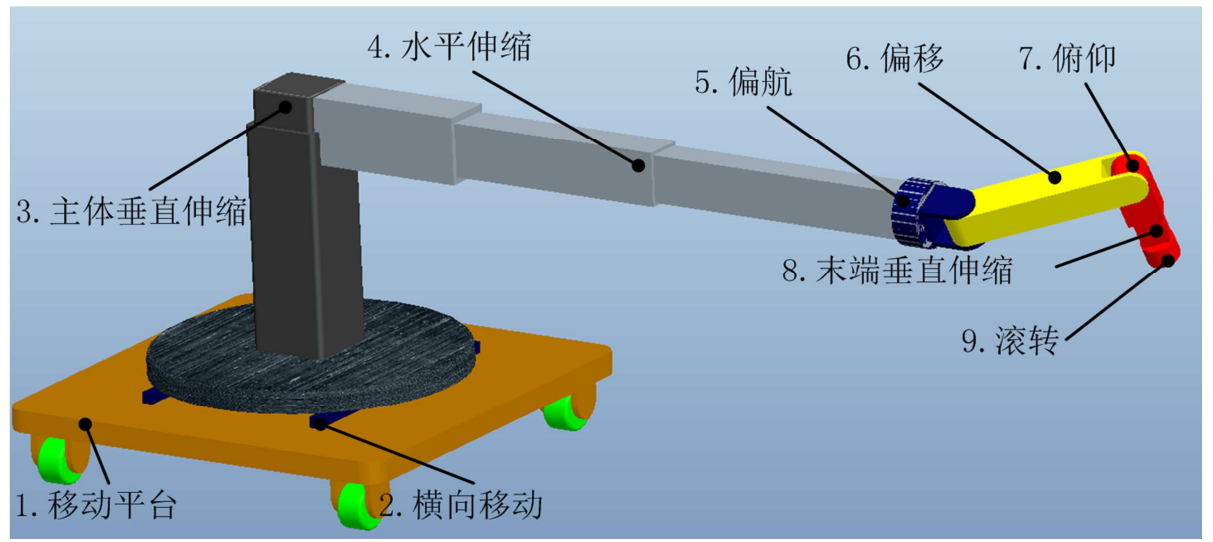

图1 多自由度装配系统构成。

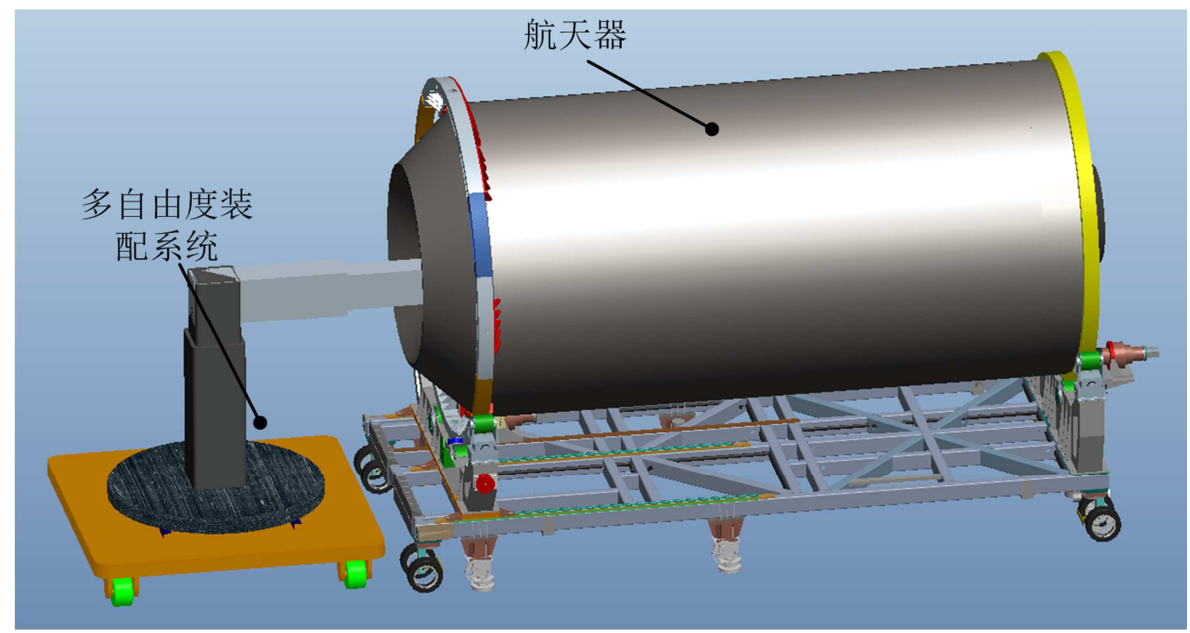

图2 多自由度装配系统与航天器组合状态。 


\section{3. 运动学模型}

空间站多自由度装配系统的姿态可以采用齐次变换 法来描述, 建立各个运动环节之间坐标系的转换与末端的 映射, 通过矩阵变换的形式将各个运动环节联系起来, 然 后通过运动分析, 建立空间站多自由度装配系统的运动模 型, 采用逆运动关系解算进行空间站多自由度装配系统的 路径规划。

\section{1. 姿态分析}

空间站多自由度装配系统内部结构及负载产品均可 以看作是刚体。因此, 各个机构的运动可以看作是刚体之 间的运动。由于当空间站多自由度装配系统运动到航天器 指定位置固定后, 这样就可以将航天器产品的坐标位置作 为全局坐标系, 即基坐标系, 令基坐标系为 $\{\mathrm{A}\}$, 其中 $x$, $y, z$ 分别描述基坐标系为 $\{\mathrm{A}\}$ 的三个坐标轴。

\section{$\{\mathrm{A}\}$}

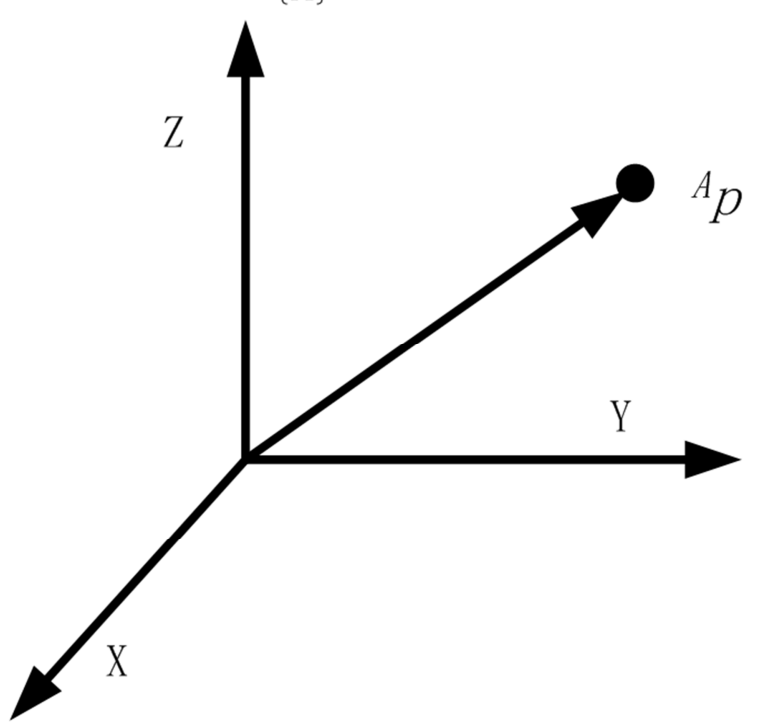

图3 基坐标系为 $\{\mathrm{A}\}$ 下任意一点p位置示意图。

当产品装配到位后，其上任意一点 $p$ 均可以采用基坐 标系为 $\{\mathrm{A}\}$ 中的一个 $3 \times 1$ 的位置矢量 ${ }^{A} p$ 来进行描述,

$$
{ }^{A} P=\left\{\begin{array}{l}
p_{x} \\
p_{y} \\
p_{z}
\end{array}\right\}
$$

其中, $p_{x}, p_{y}, p_{z}$ 分别为 $p$ 点在基坐标系为 $\{\mathrm{A}\}$ 中三个 坐标轴方向的坐标向量。

对于空间的任意位置, 为了准确表达对于基坐标系为 $\{\mathrm{A}\}$ 的位置信息, 还需要创造一个产品所在的空间坐标系, 令产品所在的空间坐标系为 $\{\mathrm{B}\}$, 产品相对于空间坐标系 为 $\{B\}$ 的位置信息已知, 通过建立空间坐标系 $\{B\}$ 相对于 基坐标系 $\{\mathrm{A}\}$ 的位置关系就可以得到任意产品在基坐标系 $\{\mathrm{A}\}$ 中的姿态。

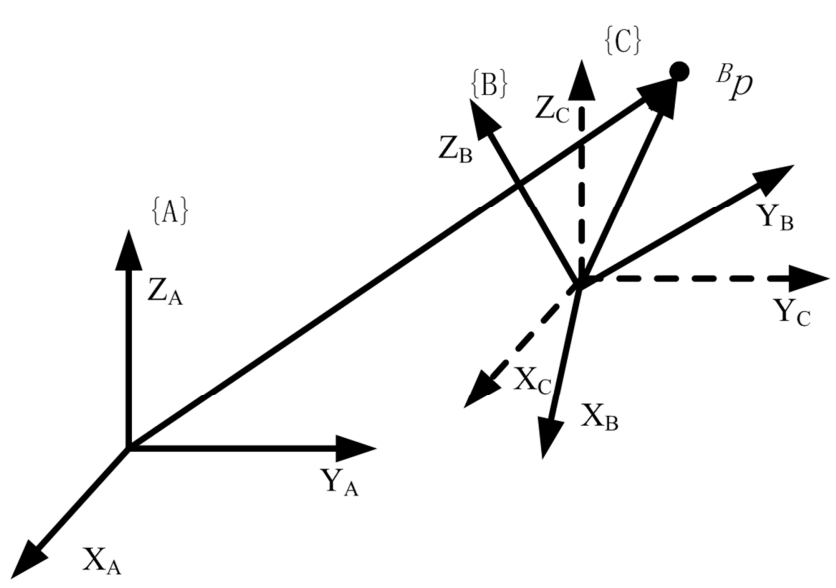

图4 基坐标系为 $\{\mathrm{A}\}$ 与空间坐标系为 $\{\mathrm{B}\}$ 的位置关系。

假设空间坐标系为 $\{\mathrm{B}\}$ 主轴方向的三个单位矢量用 $X_{B}$, $Y_{B}, Z_{B}$ 来表示, 基坐标系为 $\{\mathrm{A}\}$ 主轴方向的三个单位矢量 用 $X_{A}, Y_{A}, Z_{A}$ 来表示。通常坐标系之间既包含平移变换, 也包含旋转变换。通过空间坐标系 $\{\mathrm{B}\}$ 与基坐标系 $\{\mathrm{A}\}$ 三 个单位坐标向量的相对关系来表达坐标系之间的旋转关 系, 称之为旋转矩阵, 用 ${ }_{B} R$ 来表示, 通过空间坐标系 $\{\mathrm{B}\}$ 与基坐标系 $\{\mathrm{A}\}$ 三个单位坐标向量的相对关系来表达坐标 系之间的位置关系, 称之为平移矩阵, 用 ${ }_{B} P$ 来表示。

$$
\begin{gathered}
{ }_{B}^{A} P=\left[\begin{array}{lll}
{ }^{A} X_{B} & { }^{A} Y_{B} & { }^{A} Z_{B}
\end{array}\right] \\
{ }_{B}^{A} R=\left[\begin{array}{ccc}
X_{B} \cdot X_{A} & Y_{B} \cdot X_{A} & Z_{B} \cdot X_{A} \\
X_{B} \cdot Y_{A} & Y_{B} \cdot Y_{A} & Z_{B} \cdot Y_{A} \\
X_{B} \cdot Z_{A} & Y_{B} \cdot Z_{A} & Z_{B} \cdot Z_{A}
\end{array}\right]
\end{gathered}
$$

对于既包含平移变换, 也包含旋转变换的坐标系之间 的关系又称为复合变换, 在复合变换的情况下, 可以假定 存在中间坐标系 $\{C\}$, 令坐标系 $\{C\}$ 与坐标系 $\{B\}$ 在坐标原 点处重合, 坐标系 $\{C\}$ 与坐标系 $\{A\}$ 坐标轴方向一致, 如 图4所示。那么, 可以认为坐标系 $\{\mathrm{B}\}$ 与坐标系 $\{\mathrm{A}\}$ 之间的 变换关系为坐标系 $\{A\}$ 先经过平移得到坐标系 $\{C\}$, 然后 坐标系 $\{C\}$ 通过旋转得到坐标系 $\{B\}$, 则可以得到坐标系 $\{B\}$ 与坐标系 $\{A\}$ 之间的变换关系如式 (4) 所示:

$$
{ }^{A} P={ }^{C} P+{ }_{C}^{A} P={ }_{B}^{C} R \cdot{ }^{B} P+{ }_{B}^{A} P={ }_{B}^{A} R \cdot{ }^{B} P+{ }_{B}^{A} P
$$

将复合变换关系转化为其次变换的形式[7] [10], 可 得:

$$
\left(\begin{array}{l}
{ }^{A} P \\
1
\end{array}\right)=\left(\begin{array}{cc}
{ }_{B}^{A} R & { }_{B}^{A} P \\
0 & 1
\end{array}\right)\left(\begin{array}{l}
{ }^{B} P \\
1
\end{array}\right)
$$

\section{2. 运动模型建立}

\subsection{1. 连杆机械臂齐次变换说明}

空间站多自由度装配系统运动模型建立可以参考连 杆机械臂的齐次变换。每一个关节均可以采用连杆形式描 
述, 包含 2 个参数, 即公共法线距离 $a_{i}$ 和垂直 $a_{i}$ 所在平面的 两轴平面的扭角 $\alpha_{i}$; 需要另外两个参数来表示相邻连杆的 关系, 即两连杆的相对位置 $d_{i}$ 和两连杆支架夹角 $\theta_{i}$, 如图 5 所示。除第一个和最后一个连杆外, 每个连杆两段的轴线 各有一条法线, 分别为前、后相邻连杆的公共法线, 这两 法线间的距离为 $d_{i}$, 称 $a_{i}$ 为连杆长度, $\alpha_{i}$ 为连杆扭角, $d_{i}$ 为 两连杆距离, $\theta_{i}$ 为两连杆夹角 $[11] \sim[15]$ 。

根据上述定义, 连杆坐标系 $\{\mathrm{i}\}$ 相对于 $\{\mathrm{i}-1\}$ 的变换矩 阵称为 $T$ 矩阵, 连杆变化 $T$ 可以看成是坐标系 $\{i-1\}$ 经过如下 变换得到的; a) 连杆长度 $a_{i-1}$ : 沿着 $x_{i-1}$ 从 $z_{i-1}$ 到 $z_{i}$ 的直线距离;

b) 连杆扭角 $\alpha_{i-1}$ : 沿着 $x_{i-1}$ 从 $z_{i-1}$ 到 $z_{i}$ 的旋转角度

c) 连杆距离 $d_{i}$ : 沿着 $z_{i}$ 从 $x_{i-1}$ 到 $x_{i}$ 的直线距离;

d) 连杆夹角 $\theta_{i}$ : 沿着 $z_{i}$ 从 $x_{i-1}$ 到 $x_{i}$ 的旋转角度; 则可得:

$$
{ }_{i}^{i-1} T=\operatorname{Rot}\left(x, \alpha_{i-1}\right) \operatorname{Trans}\left(x, a_{i-1}\right) \operatorname{Rot}\left(z, \theta_{i}\right) \operatorname{Trans}\left(z, d_{i}\right)
$$

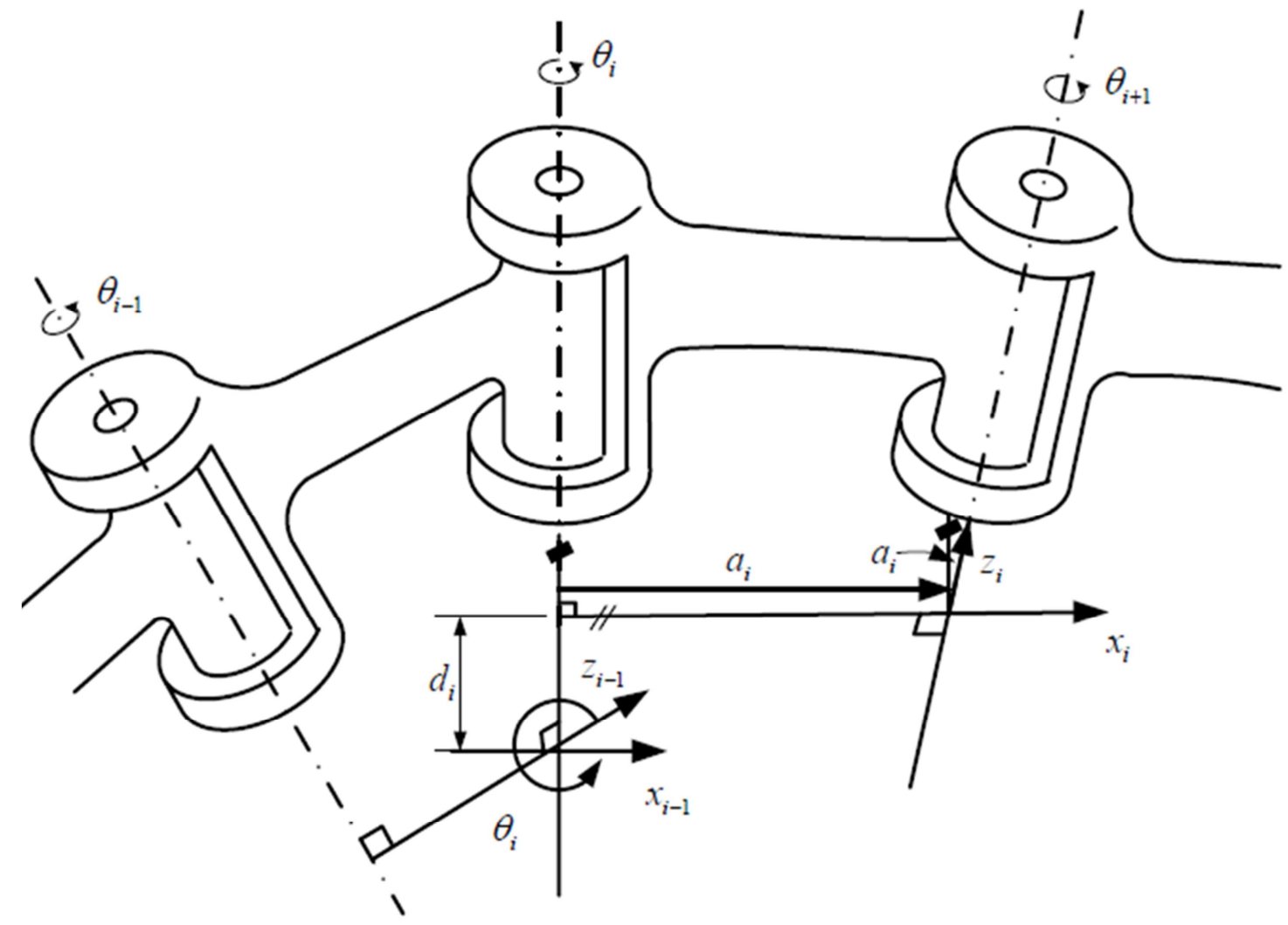

图5 连杆机械臂关节变换示意。

因此, 得到了空间站多自由度装配系统齐次变换关系, 但是在实际建立空间站多自由度装配系统在进行负载产 品安装的运动模型过程中, 为了更清晰的表达出各个运动 环节的真实运动方向, 对空间站多自由度装配系统模型进 行简化，具体如下:

a) 保持系统内部各个机构坐标系相对本身固定;

b) 坐标系z轴垂直于连杆的上表面, 沿自身垂直方向运 动;

c) 坐标系Y轴沿着结构伸长方向;

d) 对每一个机构均采用旋转与移动两种方式进行表达。

\subsection{2. 模型建立}

为了准确的描述空间站多自由度装配系统在进行负 载产品安装时的运动模型, 以航天器、待安装产品以及多 自由度装配系统装配三要素为基础, 空间站多自由度装配
系统携带负载运动到航天器指定位置为最终状态, 确定末 端负载产品相对于航天器坐标系下的运动模型。

首先, 当空间站多自由度装配系统携带产品运动到航 天器指定位置时, 则航天器相对于多自由度装配系统位置 就相对固定, 同时末端机构相对于负载产品也相对固定, 其坐标系相对关系已知, 那么, 再找到空间站多自由度装 配系统本身内部运动关系, 即可建立空间站多自由度装配 系统的运动模型。

空间站多自由度装配系统自身共包含 8 个自由度, 每 个自由度均包含一个连接环节, 可以在每一个环节位置建 立一个坐标系, 用以描述各个环节之间的位置变换关系。 将航天器产品的基坐标系记为 $\{0\}$, 空间站多自由度装配 系统的基坐标系记为 $\{1\}$, 各个连接环节的坐标系记为 $\{i\}$ （其中 $\mathrm{i}=2,3,4,5,6,7,8,9$ ）, 则将空间站多自由度装配系 统进行模型简化, 如图6所示。 


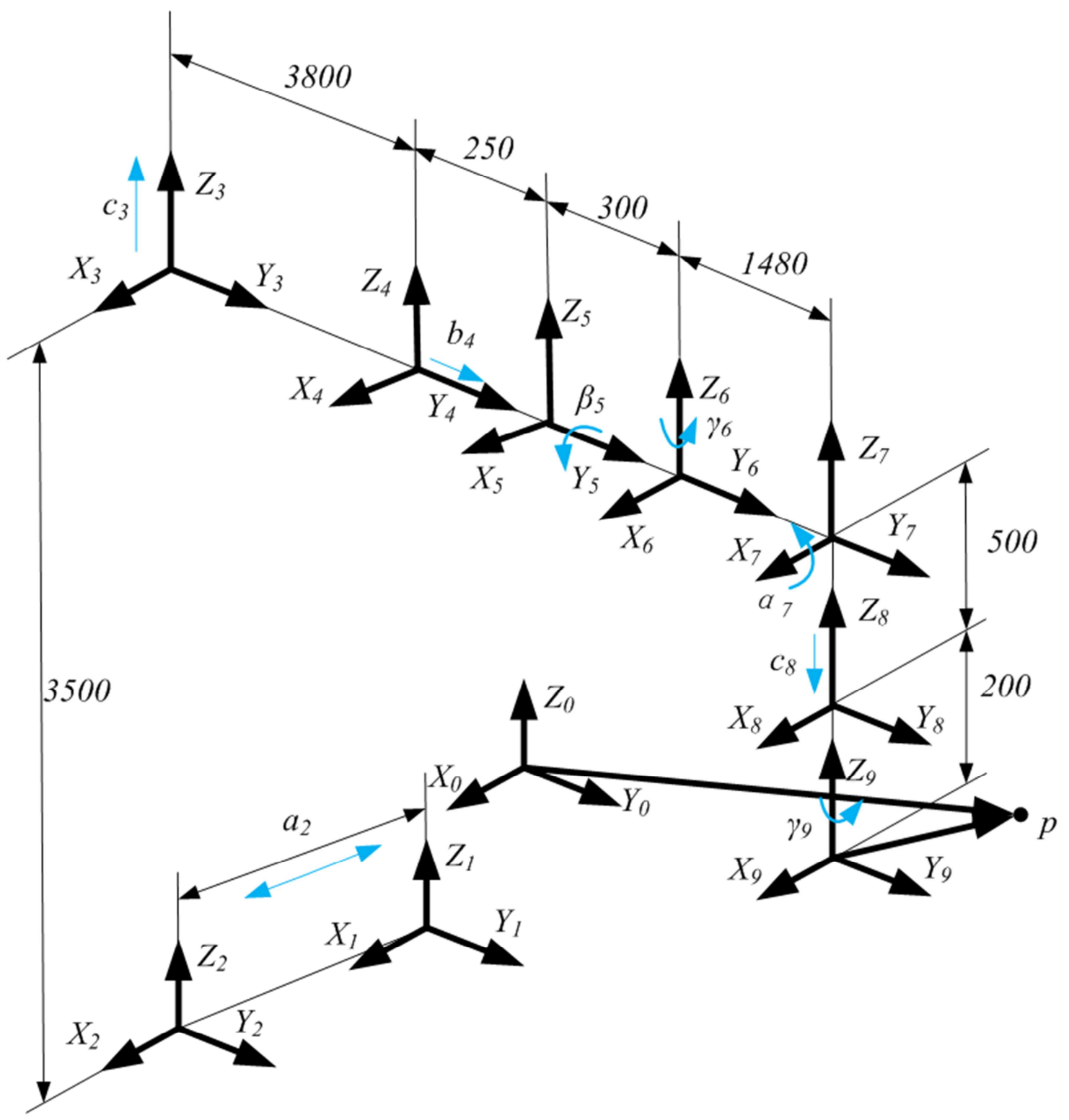

图6 空间站多自由度装配系统坐标系示意。

在建立空间站多自由度装配系统坐标系关联的过程, 为了简化模型, 对坐规定标系之间的参数进行了规定:

a) $\alpha_{i}$ : 绕 $X_{i-1}$ 到 $X_{i}$ 轴旋转角度;

b) $\beta_{i}$ : 绕 $Y_{i-1}$ 到 $Y_{i}$ 轴旋转角度;

c) $\gamma_{i}$ : 绕 $Z_{i-1}$ 到 $Z_{i}$ 轴旋转角度;

d) $a_{i}:$ 沿着 $X_{i-1}$ 移动距离; e) $b_{i}$ : 沿着 $Y_{i-1}$ 移动距离;

f) $c_{i}:$ 沿着 $Z_{i-1}$ 移动距离;

依据航天器、负载产品、空间站多自由度装配系统模 型坐标系可以得到各个运动环节之间的相对位置关系, 各 个坐标系之间的相对位置关系如表1所示。

表1 运动环节之间的相对关系说明。

\begin{tabular}{llllllll}
\hline 坐标系 & $\boldsymbol{\alpha}_{\boldsymbol{i}}$ & $\boldsymbol{\beta}_{\boldsymbol{i}}$ & $\gamma_{\boldsymbol{i}}$ & $\boldsymbol{a}_{\boldsymbol{i}}$ & $\boldsymbol{b}_{\boldsymbol{i}}$ & $\boldsymbol{c}_{\boldsymbol{i}}$ & 移动与转动范围 \\
\hline 坐标系 $\{1\}$ & 0 & 0 & 0 & 0 & -2500 & -3800 & $/$ \\
坐标系 $\{2\}$ & 0 & 0 & 0 & $a_{2}$ & 0 & 0 & $-1000 \sim+1000$ \\
坐标系 $\{3\}$ & 0 & 0 & 0 & 0 & 0 & $3500+c_{3}$ & $0 \sim 2000$ \\
坐标系 $\{4\}$ & 0 & 0 & 0 & 0 & $3800+b_{4}$ & 0 & $0 \sim 3000$ \\
坐标系 $\{5\}$ & 0 & $\beta_{5}$ & 0 & 0 & 250 & 0 & $-180^{\circ} \sim+180^{\circ}$ \\
坐标系 $\{6\}$ & 0 & 0 & $\gamma_{6}$ & 0 & 300 & 0 & $-90^{\circ} \sim+90^{\circ}$ \\
坐标系 $\{7\}$ & $\alpha_{7}$ & 0 & 0 & 0 & 1480 & 0 & $-45^{\circ} \sim+90^{\circ}$ \\
坐标系 $\{8\}$ & 0 & 0 & 0 & 0 & 0 & $-500-c_{8}$ & $0 \sim 500$ \\
坐标系 $\{9\}$ & 0 & 0 & $\gamma_{9}$ & 0 & 0 & -200 & $-180^{\circ} \sim+180^{\circ}$ \\
\hline
\end{tabular}


根据各个运动环节的坐标系关系以及任意物体的姿态分析就可以得到不同坐标系下的转换矩阵, 最终就可以求得 末端负载坐标系相对于航天器产品的坐标系转换矩阵。

令 ${ }_{i}^{i-1} T$ 代表坐标系 $\{\mathrm{i}\}$ 相对于 $\{\mathrm{i}-1\}$ 之间的坐标变换, 则末端负载坐标系相对于航天器产品的坐标系转换矩阵为:

$$
\begin{aligned}
& { }_{9}^{0} T={ }_{9}^{8} T_{8}^{7} T \cdot{ }_{7}^{6} T \cdot{ }_{6}^{5} T \cdot{ }_{5}^{4} T \cdot{ }_{4}^{3} T \cdot{ }_{3}^{2} T \cdot{ }_{2}^{1} T \cdot{ }_{1}^{0} T \\
& =\left(\begin{array}{cccc}
r 11 & r 12 & r 13 & r 14 \\
r 21 & r 22 & r 23 & r 24 \\
r 31 & r 32 & r 33 & r 34 \\
0 & 0 & 0 & 1
\end{array}\right)
\end{aligned}
$$

其中式中的各个参数具体代表如下:

$$
\begin{aligned}
& r 11=\sin \left(\gamma_{9}\right) *\left(\sin \left(\alpha_{7}\right) * \sin \left(\beta_{5}\right)-\cos \left(\alpha_{7}\right) * \cos \left(\beta_{5}\right) * \sin \left(\gamma_{6}\right)\right) \\
& +\cos \left(\beta_{5}\right) * \cos \left(\gamma_{6}\right) * \cos \left(\gamma_{9}\right) \\
& r 12=\cos \left(\gamma_{9}\right) *\left(\sin \left(\alpha_{7}\right) * \sin \left(\beta_{5}\right)-\cos \left(\alpha_{7}\right) * \cos \left(\beta_{5}\right) * \sin \left(\gamma_{6}\right)\right) \\
& -\cos \left(\beta_{5}\right) * \cos \left(\gamma_{6}\right) * \sin \left(\gamma_{9}\right) \\
& r 13=\cos \left(\alpha_{7}\right) * \sin \left(\beta_{5}\right)+\cos \left(\beta_{5}\right) * \sin \left(\alpha_{7}\right) * \sin \left(\gamma_{6}\right) \\
& r 14=a_{2}-200 * \cos \left(\alpha_{7}\right) * \sin \left(\beta_{5}\right)-1480 * \cos \left(\beta_{5}\right) * \sin \left(\gamma_{6}\right) \\
& -\left(\cos \left(\alpha_{7}\right) * \sin \left(\beta_{5}\right)+\cos \left(\beta_{5}\right) * \sin \left(\alpha_{7}\right) * \sin \left(\gamma_{6}\right)\right) \\
& *\left(c_{8}+500\right)-200 * \cos \left(\beta_{5}\right) * \sin \left(\alpha_{7}\right) * \sin \left(\gamma_{6}\right) \\
& r 21=\cos \left(\gamma_{9}\right) * \sin \left(\gamma_{6}\right)+\cos \left(\alpha_{7}\right) * \cos \left(\gamma_{6}\right) * \sin \left(\gamma_{9}\right) \\
& r 22=\cos \left(\alpha_{7}\right) * \cos \left(\gamma_{6}\right) * \cos \left(\gamma_{9}\right)-\sin \left(\gamma_{6}\right) * \sin \left(\gamma_{9}\right) \\
& r 23=-\cos \left(\gamma_{6}\right) * \sin \left(\alpha_{7}\right) \\
& r 24=b_{4}+1480 * \cos \left(\gamma_{6}\right)+200 * \cos \left(\gamma_{6}\right) * \sin \left(\alpha_{7}\right) \\
& +\cos \left(\gamma_{6}\right) * \sin \left(\alpha_{7}\right) *\left(c_{8}+500\right)+1850 \\
& r 31=\sin \left(\gamma_{9}\right) *\left(\cos \left(\beta_{5}\right) * \sin \left(\alpha_{7}\right)+\cos \left(\alpha_{7}\right) * \sin \left(\beta_{5}\right) * \sin \left(\gamma_{6}\right)\right) \\
& -\cos \left(\gamma_{6}\right) * \cos \left(\gamma_{9}\right) * \sin \left(\beta_{5}\right) \\
& r 32=\cos \left(\gamma_{9}\right) *\left(\cos \left(\beta_{5}\right) * \sin \left(\alpha_{7}\right)+\cos \left(\alpha_{7}\right) * \sin \left(\beta_{5}\right) * \sin \left(\gamma_{6}\right)\right) \\
& +\cos \left(\gamma_{6}\right) * \sin \left(\beta_{5}\right) * \sin \left(\gamma_{9}\right) \\
& r 33=\cos \left(\alpha_{7}\right) * \cos \left(\beta_{5}\right)-\sin \left(\alpha_{7}\right) * \sin \left(\beta_{5}\right) * \sin \left(\gamma_{6}\right) \\
& r 34=c_{3}-200 * \cos \left(\alpha_{7}\right) * \cos \left(\beta_{5}\right)+1480 * \sin \left(\beta_{5}\right) * \sin \left(\gamma_{6}\right) \\
& -\left(\cos \left(\alpha_{7}\right) * \cos \left(\beta_{5}\right)-\sin \left(\alpha_{7}\right) * \sin \left(\beta_{5}\right) * \sin \left(\gamma_{6}\right)\right) *\left(c_{8}+500\right) \\
& +200 * \sin \left(\alpha_{7}\right) * \sin \left(\beta_{5}\right) * \sin \left(\gamma_{6}\right)-300 \\
& \left.r y_{6}\right)
\end{aligned}
$$

由上述矩阵 ${ }_{9}^{0} T$, 当已知负载产品相对于末端坐标系的位置关系, 通过解算空间站多自由度装配系统自身的转换矩 阵 $_{9}{ }_{9} T$, 就可以得到负载产品相对于航天器基坐标系下的位置关系。

为了验证转换矩阵 ${ }_{9} T$ 的正确性, 设定一组空间站多自由度装配系统各个机构的参数, 假定末端负载就位于 $\{9\}$ 的 坐标原点, 然后通过理论计算及模型测量来验证转换矩阵 ${ }_{9}^{0} T$ 的正确性。假定参数如下:

$$
a_{2}=0, \quad c_{3}=700, b_{4}=1900, \quad c_{8}=250, \quad \beta_{5}=0, \quad \gamma_{6}=-30^{\circ}, \quad \alpha_{7}=0, \gamma_{9}=0 \text { 。 }
$$


经过计算得:

$$
\begin{aligned}
& { }^{0} P=\left(\begin{array}{cccc}
r 11 & r 12 & r 13 & r 14 \\
r 21 & r 22 & r 23 & r 24 \\
r 31 & r 32 & r 33 & r 34 \\
0 & 0 & 0 & 1
\end{array}\right) \cdot\left(\begin{array}{l}
0 \\
0 \\
0 \\
1
\end{array}\right)= \\
& \left(\begin{array}{cccc}
0.866 & 0.5 & 0 & 740 \\
-0.5 & 0.866 & 0 & 5031.72 \\
0 & 0 & 1 & -550 \\
0 & 0 & 0 & 1
\end{array}\right) \cdot\left(\begin{array}{l}
0 \\
0 \\
0 \\
1
\end{array}\right)=\left(\begin{array}{l}
740 \\
5031.72 \\
-550 \\
1
\end{array}\right)
\end{aligned}
$$

通过模型测量末端坐标原点相对于航天器基坐标系 的位置, 得到坐标为 $[740,5031.72,-550]$, 经过计算分 析与模型测量比较, 理论推导转换矩阵得到的结果与实测 结果是一致的, 从而验证了转换矩阵的正确性。

\section{3. 逆运动路径分析}

在实际操作过程中, 需要根据负载产品的安装位置来 调节空间站多自由度装配系统内部机构调节参数, 使其满 足产品安装需求。因此, 此问题可以看作是空间站多自由 度装配系统的逆运动问题, 已知负载产品最终相对于末端 以及航天器基坐标系的位置关系, 求解空间站多自由度装 配系统各个调节机构的运动参数。

为了便于空间站多自由度系统内部机构参数的求解, 需要对系统运动进行简化:

a) 由于空间站多自由度装配系统包含了两个垂直向的 自由度, 这两个自由度是重合的, 为了便于计算, 在参数确立的过程中, 假定末端垂直伸缩不进行运 动, 即 $c_{8}=0$, 将全部垂直向运动转化为主体垂直伸 缩处, 然后根据实际运动过程在距离物体垂直表面 $500 \mathrm{~mm}$ 以内位置开始启动末端垂直伸缩运动。

b) 待空间站多自由度装配系统携带产品运送到舱内指定 区域时, 首先将末端机构 6 , 即绕Z轴回转机构进行调 节, 使其转动到X向距离负载 $200 \mathrm{~mm}$ 位置, 然后通过调 节俯仰、偏航 3 个调节机构来实现将末端产品调整到表 面与安装面角度一致 (注: 仅限于有角度安装的产品)。

c) 通过主体垂直伸缩、水平伸缩来进行产品位置调节, 然后通过末端垂直伸缩实现产品装配, 对于复杂的 多角度产品装配还需要调节横向移动机构, 避免由 于末端垂直伸缩行程不足导致无法装配。

针对空间站多自由度装配系统逆运动路径求解过程, 基于以上假设, 可以根据航天器产品的实际装配过程, 将 装配分为两种工况进行考虑。

a) 产品安装过程不需要通过俯仰、偏航机构进行角度 调节;

b) 产品为复杂多角度安装状态, 需要通过俯仰、偏航 机构进行角度调节。

\subsection{1. 无角度调节状态}

航天器大部分产品其安装面均为与航天器基坐标平 面平行状态, 此时可以存在: $a_{2}=0, \beta_{5}=0, \alpha_{7}=0, c_{8}=300$, $\gamma_{9}$ 为 $0^{\circ}$ 或 $-90^{\circ}$ 或 $+90^{\circ}$ 。此时空间站多自由度装配系统内部 转换矩阵 ${ }_{9} T T$ 可以简化为:

$$
\begin{aligned}
& r 11=\cos \left(\gamma_{6}\right) * \cos \left(\gamma_{9}\right)-\sin \left(\gamma_{6}\right) * \sin \left(\gamma_{9}\right) \\
& r 12=-\cos \left(\gamma_{6}\right) * \sin \left(\gamma_{9}\right)-\cos \left(\gamma_{9}\right) * \sin \left(\gamma_{6}\right) \\
& r 13=0 \\
& r 14=-1480 * \sin \left(\gamma_{6}\right) \\
& r 21=\cos \left(\gamma_{6}\right) * \sin \left(\gamma_{9}\right)+\cos \left(\gamma_{9}\right) * \sin \left(\gamma_{6}\right) \\
& r 22=\cos \left(\gamma_{6}\right) * \cos \left(\gamma_{9}\right)-\sin \left(\gamma_{6}\right) * \sin \left(\gamma_{9}\right) \\
& r 23=0 \\
& r 24=b_{4}+1480 * \cos \left(\gamma_{6}\right)+1850 \\
& r 31=0 \\
& r 32=0 \\
& r 33=1 \\
& r 34=c_{3}-1300
\end{aligned}
$$

首先, 根据末端坐标系原点所在航天器基坐标系的位 置就可以解算出 $\gamma_{6}, c_{3}, b_{4}$ 的数值, 然后通过产品安装面 与航天器基坐标平面的位置关系就可以得到转动的角度 $\gamma_{9}$, 从而计算出了空间站多自由度装配系统各个机构的调节 参数。

\subsection{2. 有角度调节状态}

航天器部分产品的安装面与航天器基坐标平面存在 一定的角度; 此时空间站多自由度装配系统坐标系转换关 系见式7。

首先使系统的偏移机构 6 转动到 $\mathrm{X}$ 向距离负载 $200 \mathrm{~mm}$ 位置, 即 $\gamma_{6}$ 已知, 假设末端平面与航天器基坐标平面角度 余弦值分别为 $p 1, p 2, p 2$, 此时可以根据坐标关系确定 $\gamma_{6}$, 则, 将上述转换矩阵中的 ${ }_{5} T,{ }_{6}^{5} T,{ }_{7}^{6} T$ 进行相乘得到末端 平面相对航天器基坐标平面的相对角度, 进而求解得到 $\beta_{5}$, $\alpha_{7}$, 得到角度调节参数。

$$
\beta_{5}=\operatorname{asin}\left(p 2 / \cos \left(\gamma_{6}\right)\right)
$$

然后旋转末端滚转机构, 将末端产品安装面与航天器 安装面相同, 得到 $\gamma_{9}$ 。

令 $c_{8}=300$, 调节主体伸缩机构、水平伸缩机构、横向 移动机构来计算得到 $a_{2}, c_{3}, b_{4}$ 。最后, 通过末端伸缩机 构实现产品的精确装配。

\section{4. 仿真分析}

由于无角度调节状态比较简单, 通过知道末端位置就 可以直接求解出空间站多自由度装配系统内部机构调节 参数, 本文仅对多角度调节状态进行仿真分析。

首先已知产品安装位置及与系统末端连接位置, 则可 以得到末端与航天器基坐标平面的角度关系: $\alpha, \beta, \gamma$; 与系统末端连接位置, 即末端坐标原点处的尺寸: $x, y$, $z$; 同时知道末端相对于产品的轴向距离 $a, b, c$, 距离产 品与末端连接位置垂直向距离 $300 \mathrm{~mm}$ 位置的坐标 $x^{c}, y^{c}, z^{\prime}$ 。 
则可以将空间站多自由度装配系统的调节参数进行如下 优化分解:

目标: 空间站多自由度装配系统实际到达位置与给定 位置的角度和位置偏差最小。

约束 $1: 1480^{*} \gamma_{6}=\mathrm{a}$; 另外两个角度调节为离散值取 [-2:0.1:2], 精度为 $0.1^{\circ}$, 逐次逼近得到末端角度 $\beta_{5}, \alpha_{7}$;

约束 2 : 通过 $\gamma_{9}$ 取离散值取 [-2:0.1:2], 精度为 $0.1^{\circ}$, 逐 次逼近得到末端角度 $\gamma_{9}$;

约束 3: 通过 $c_{8}=300$; 首先将位置调节至此位置附近, 然后将外尺寸调节取 [-2:1:2], 精度为 $0.1 \mathrm{~mm}$, 逐次逼近得 到 $a_{2}, c_{3}, b_{4}$;

约束 4 :

$$
\left\{\begin{array}{c}
-1000 \leq a_{2} \leq 1000 \\
0 \leq c_{3} \leq 2000 \\
0 \leq b_{4} \leq 3000 \\
-180^{\circ} \leq \beta_{5} \leq 180^{\circ} \\
-90^{\circ} \leq \gamma_{6} \leq 90^{\circ} \\
-45^{\circ} \leq \alpha_{7} \leq 90^{\circ} \\
c_{8}=300 \\
-180^{\circ} \leq \gamma_{9} \leq 180^{\circ}
\end{array}\right.
$$

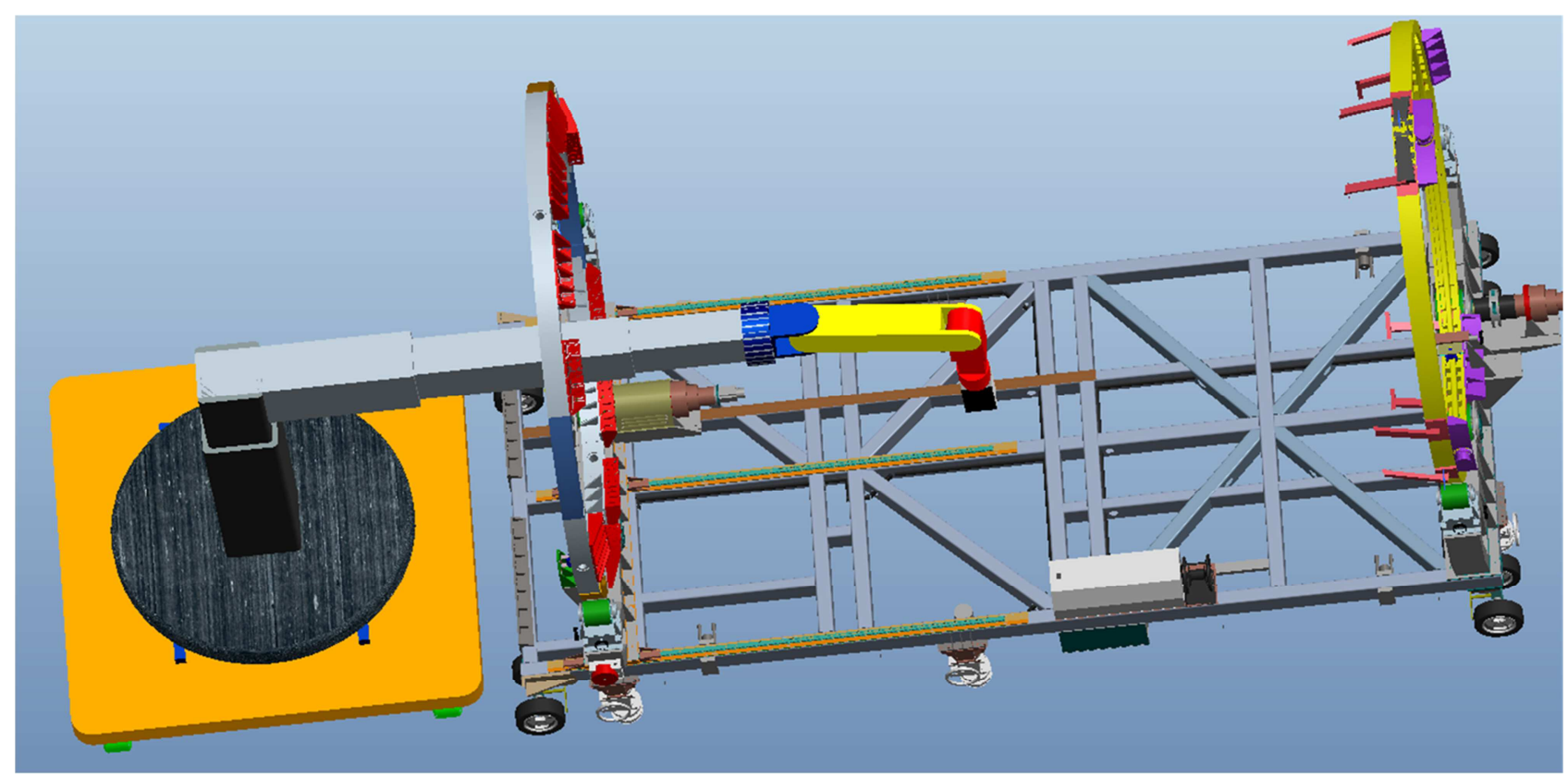

图7 无角度调节产品安装示意图。

\section{2. 有角度调节产品装配}

在航天器内部选取需要通过俯仰、偏航机构进行角度调节的产品Q, 产品安装状态如图8所示
则依据上述约束条件及优化后得到的目标, 就可以将 问题转换为仿真求解过程, 通过多次不断迭代就可以得到 最终求得仿真结果。

\section{4. 装配模拟及仿真验证}

针对得到的空间站多自由度装配系统逆运动分析结 果, 选取典型的产品进行模拟装配过程, 验证理论模型结 果的正确性。

\section{1. 无角度调节产品装配}

在航天器内部选取不需要通过俯仰、偏航机构进行角 度调节的产品P, 产品P安装面与航天器基坐标平面ZX面 平行, 同时末端机构直接与产品进行连接装配, 产品安装 状态如图7所示。

则首先令 $a_{2}=0, \beta_{5}=0, \alpha_{7}=0, c_{8}=300, \gamma_{9}$ 为 $0^{\circ}$, 然后根 据3.3.1节内容求得 $\gamma_{6}=6.148, c_{3}=111, b_{4}=944.63$, 进而得 到了空间站多自由度装配系统此状态下的转换矩阵 ${ }_{9}{ }_{9} T$ 。计 算得到末端的坐标原点坐标为 $[158.5,4266.1,-1189]$ 。

通过末端的坐标原点来验证理论结果的正确性, 模型 测量结果为 $[158.5,4266.1,-1189]$, 理论计算结果与模型 测量结果一致, 证明理论推导模型是正确性。 


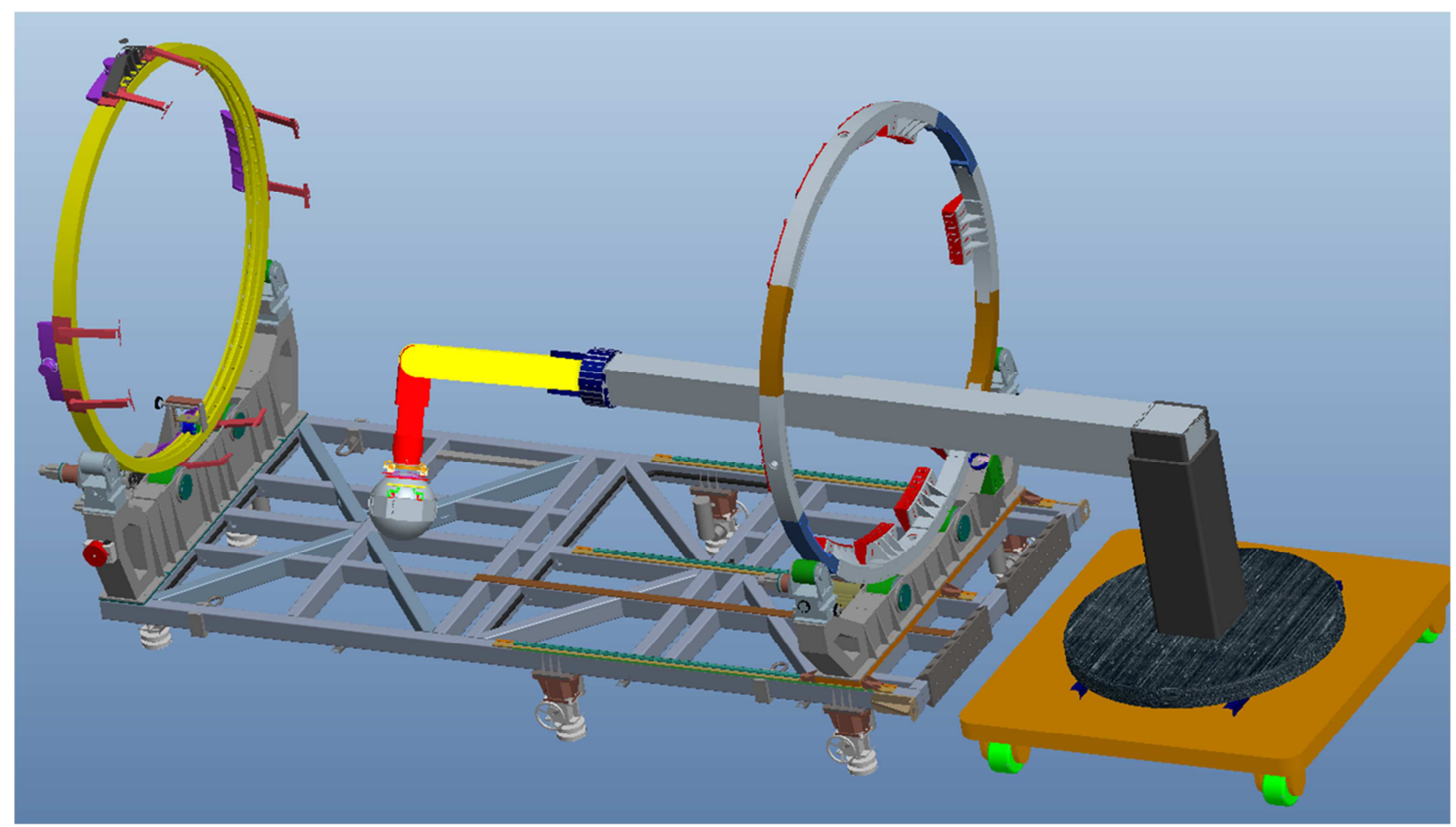

图8 有角度调节产品安装示意图。

按照3.3.2节分析, 首先使系统的偏移机构 6 转动到 $\mathrm{X}$ 向距离负载 $200 \mathrm{~mm}$ 位置, 则计算得到 $\gamma_{6}=29.36^{\circ}$, 通过角度 转换关系进而求解得到 $\beta_{5}=-27.47^{\circ}, \alpha_{7}=8.69^{\circ}$ 。

然后旋转末端滚转机构, 将末端产品安装面与航天器 安装面相同, 得到 $\gamma_{9}=25.83^{\circ}$

令 $c_{8}=300$, 调节主体伸缩机构、水平伸缩机构、横向 移动机构来调节位置参数, 计算得到 $a_{2}=-197.35, c_{3}=505.69$, $b_{4}=2398.62$ 。得到空间站多自由度装配系统运动参数后, 就可以计算得到末端的坐标原点坐标为 $[-450.91,5670.21$, -1040.27]。

通过仿真分析: 得到偏移机构转角 $\gamma_{6}=29.36^{\circ}$; 通过平 面之间角度转换关系进而求解得到偏航和俯仰, 即 $\beta_{5}=-27.5^{\circ}, \alpha_{7}=8.7^{\circ}$; 通过末端滚转机构分析, 得到 $\gamma_{9}=25.8^{\circ}$; 最后, 当 $c_{8}=300$, 通过坐标位置关系得到主体伸缩机构、 水平伸缩机构、横向移动机构调节参数, 即 $a_{2}=-197.9$, $c_{3}=505.7, b_{4}=2398.6$ 。则计算得到末端的坐标原点坐标为 [-450.9, 5670.34, -1040.41]。

模型测量结果为 $[-450.91,5670.21,-1040.27]$, 将模 型测量结果与计算结果及仿真分析结果进行比较, 如表2 所示, 通过表 2 可以看出, 理论计算结果与模型测量是一 致的, 而仿真结果由于选取的精度问题, 导致存在一定的 误差, 最大的误差出现在产品 Z轴方向, 最大偏差为 $0.14 \mathrm{~mm}$, 这个误差在实际使用偏差允许范围之内, 同样 满足使用要求, 可以用于指导空间站多自由度装配系统安 装产品。

\section{5. 结论}

为了满足航天器智能制造的需求, 空间站项目研制了 多自由度装配系统实现密封舱内大型产品的智能装配。本
文通过对航天器、待安装产品以及多自由度装配系统进行 分析, 对产品在航天器装配过程的姿态进行描述和分析, 建立了多自由度装配系统的运动模型, 详细分析了多自由 度装配系统的运动参数。并采用仿真软件对多自由度装配 系统的运动轨迹及路径进行模拟, 验证了理论模型及计算 结果。可以有效地指导空间站项目研制了多自由度装配系 统的使用。

\section{致谢}

本文为航天装备预研项目 《数字化装配技术》 (41423010401)的阶段性成果之一。

\section{参考文献}

[1] 谭维炽,胡金刚.航天器系统工程[M].北京:中国科学技术出 版社,2009。

[2] 吕景辉,刘玉刚.航天器装配定位机构的设计与实现[J].航天 器环境工程.2012,29(4):464-467。

[3] 韩兴龙, 李曼丽, 赵永平, 张旭明, 周乐, 航天器总装MES系统 的设计及关键技术 [J].航天制造技术.2015(4):55-59。

[4] 周建平. 我国空间站工程总体构想 [J]. 载人航 天.2013,19(2):1-10。

[5] 王永志.实施我国载人空间站工程推动载人航天事业科学

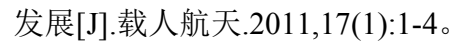

[6] 李大明,饶炜,胡成威, 空间站机械臂关键技术研究[J].载人航 天.2014(3):238-242。 
[7] 王帅军.模块化机械臂设计及其路径规划方法研究[D].哈尔 滨:哈尔滨工业大学, 2015。

[8] Wang Li-Jun, Zhou Jian-Bin, Xing Long, Zhang Shen-Feng. Numerical Simulation and Space Motion Trajectory Analysis of 6R Serial Manipulators [J]. Inernational Journal of Applied Mathematics and Statistics, Int. J. Appl. Math. Stat, 2013, 51 (21):438 446.

[9] 蔡陈阳,潘晓新.坐标系变换在精密测量中的应用[J].装备制 造技术,2017(6):233-236。

[10] Liu Xinjun, Chen Xiang, Li Zhidong. Modular Design of Typical Rigid Links in Parallel Kinematic Machines:Classification and Topology Optimization[J]. Frontiers of Mechanical Engineering, 2012, 7 (2):199-209.

[11] Sun K, Liu H, Xie Z, et al. Structure Design of an End Effector for the Chinese Space Station Experimental Module
Manipulator[C]//12th International Symposium on Artifacial Intelligence, Robotics and Automation in Space, Montreal, Canada, June 17-19,2014.

[12] 侯忠坤.六自由度教学机器人手臂系统研究[D].成都: 西南 交通大学,2011。

[13] Ayten K K, Iravani P, Sahinkaya M N. Optimal Trajectory Planning for Industrial Robots through Inverse Dynamics [C]. ICINCO (1), 2011:105-110.

[14] Crane C D, Duffy J, Carnahan T. A Kinematic Analysis of the Space Station Remote Manipulator System. Journal of Robotic Systems. 1991, 8 (5): 637-658.

[15] 间继宏,郭金鍂,刘玉斌,赵杰.一种模块化机械臂的设计与运动 学分析[J].哈尔滨工业大学学报,2015,47(1):20-25。 